\title{
Large-Scale Location-Aware Services in Access: Hierarchical Building/Floor Classification and Location Estimation using Wi-Fi Fingerprinting Based on Deep Neural Networks
}

\author{
Kyeong Soo Kim*, Ruihao Wang*, Zhenghang Zhong*, Zikun Tan*, Haowei Song ${ }^{\dagger}$, Jaehoon Cha*, and Sanghyuk Lee* \\ ${ }^{*}$ Department of Electrical and Electronic Engineering \\ Xi' an Jiaotong-Liverpool University \\ Suzhou, 215123, P. R. China \\ ${ }^{\dagger}$ Department of Computer Science and Software Engineering \\ Xi'an Jiaotong-Liverpool University \\ Suzhou, 215123, P. R. China
}

\begin{abstract}
One of key technologies for future large-scale location-aware services in access is a scalable indoor localization technique. In this paper, we report preliminary results from our investigation on the use of deep neural networks (DNNs) for hierarchical building/floor classification and floor-level location estimation based on Wi-Fi fingerprinting, which we carried out as part of a feasibility study project on Xi'an Jiaotong-Liverpool University (XJTLU) Campus Information and Visitor Service System. To take into account the hierarchical nature of the building/floor classification problem, we propose a new DNN architecture based on a stacked autoencoder for the reduction of feature space dimension and a feed-forward classifier for multi-label classification with $\arg \max$ functions to convert multi-label classification results into multi-class classification ones. We also describe the demonstration of a prototype DNN-based indoor localization system for floor-level location estimation using real received signal strength (RSS) data collected at one of the buildings on the XJTLU campus. The preliminary results for both building/floor classification and floor-level location estimation clearly show the strengths of DNN-based approaches, which can provide near state-of-the-art performance with less parameter tuning and higher scalability.

Index Terms-Indoor localization, Wi-Fi fingerprinting, deep learning, neural networks, multi-label classification, multi-class classification.
\end{abstract}

\section{INTRODUCTION}

In an indoor environment where there is no line-of-sight signal from global positioning systems (GPSs), received signal strengths (RSSs) from wireless network infrastructure can be used for localization through fingerprinting: For example, a vector of a pair of a service set identifier (SSID) and an RSS for a Wi-Fi access point (AP) measured at a location becomes its location fingerprint. A position of a user/device then can be estimated by finding the closest match between its RSS measurement and the fingerprints of known locations in a database [1].

When the indoor localization is to cover a large campus or a big shopping mall where there are lots of buildings with many floors, the scalability of fingerprinting techniques becomes an important issue. The current state-of-the-art WiFi fingerprinting techniques assume a hierarchical approach to the indoor localization, where the building, floor, and position (e.g., a label or coordinates) of a location are estimated one at a time. In [2], for instance, building estimation is done by the following process: Given the AP with the strongest RSS in a measured fingerprint, we first build a subset of fingerprints where the same AP has the strongest RSS; then, we count the number of fingerprints associated to each building and set the estimated building to be the most frequent one from the counting. Similar procedures are also proposed to estimate a floor inside the building. According to the results in [2], the best building and floor hit rates achieved for the UJIIndoorLoc dataset [3] are $100 \%$ and $94 \%$, respectively.

One of the major challenges in Wi-Fi fingerprinting is how to deal with the random fluctuation of a signal, the noise from multi-path effects, and the device dependency in RSS measurements. Unlike traditional solutions relying on complex filtering and time-consuming manual parameter tuning, machine learning techniques - especially the popular deep neural networks (DNNs) - can provide attractive solutions to Wi-Fi fingerprinting due to less parameter tuning and better scalability in larger environments [4]-[6].

In this paper, we introduce a feasibility study on the $X i$ 'an Jiaotong-Liverpool University (XJTLU) Campus Information and Visitor Service System as a test bed for largescale location-aware services in access. We report preliminary results from the investigation on the use of DNNs for hierarchical building/floor classification and floor-level location estimation, which we carried out as part of this feasibility study. We also describe the demonstration of a prototype DNN-based indoor localization system for floor-level location estimation using real RSS data, which we measured at one of the buildings on the XJTLU campus. 


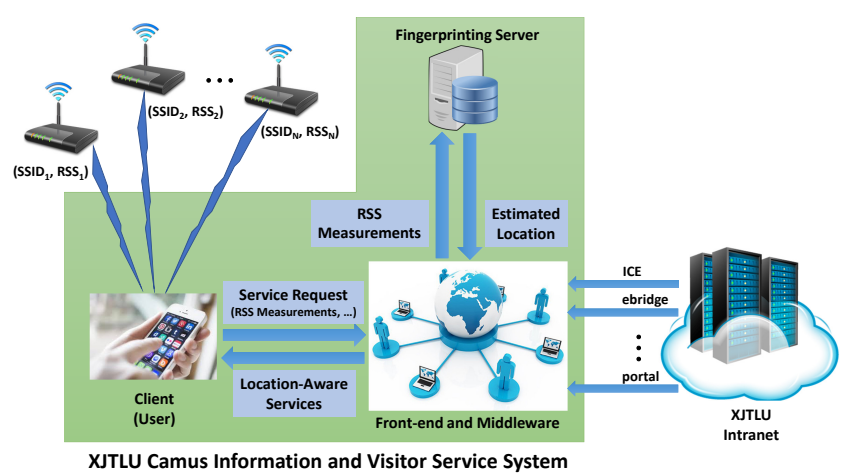

(a)
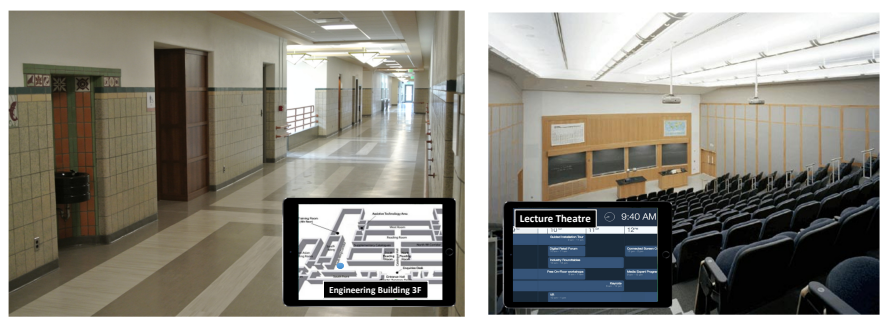

(b)

Fig. 1. XJTLU campus information and visitor service: (a) Architecture and (b) location-aware service examples.

The outline of the rest of the paper is as follows: In Sec. III. we introduce the feasibility study project on the XJTLU Campus Information and Visitor Service System. In Sec. III we discuss the hierarchical classification of building and floor based on a multi-label classifier with arg max functions. Sec. IV describes the demonstration of a prototype DNN-based indoor localization system for floor-level location estimation. Sec. V] summarizes our work described in this paper.

\section{XJTLU CAmpus Information AND Visitor SERVICE System: A TEST BED FOR LARGE-SCALE LOCATION-AWARE SERVICES IN ACCESS}

Location awareness is one of enabling technologies for future smart and green cities; understanding where people spend their times and how they interact with environments is critical to realizing this vision. At XJTLU, a group of researchers from Electrical and Electronic Engineering, Computer Science \& Software Engineering, and Urban Planning, together with an external researcher from Electrical and Electronic Engineering of City University of London, U.K., has been carrying out a feasibility assessment and road mapping for XJTLU Campus Information and Visitor Service System with the aim of identifying key component technologies and preparing plans for its implementation as a test bed for large-scale location-aware services in access and its use cases for behavioral study of students and visitors on the campus.

Fig. 1] shows an overall architecture of the XJTLU Campus Information and Visitor Service System and location aware service examples provided by the system. At the core of the system is indoor localization based on wireless fingerprinting,
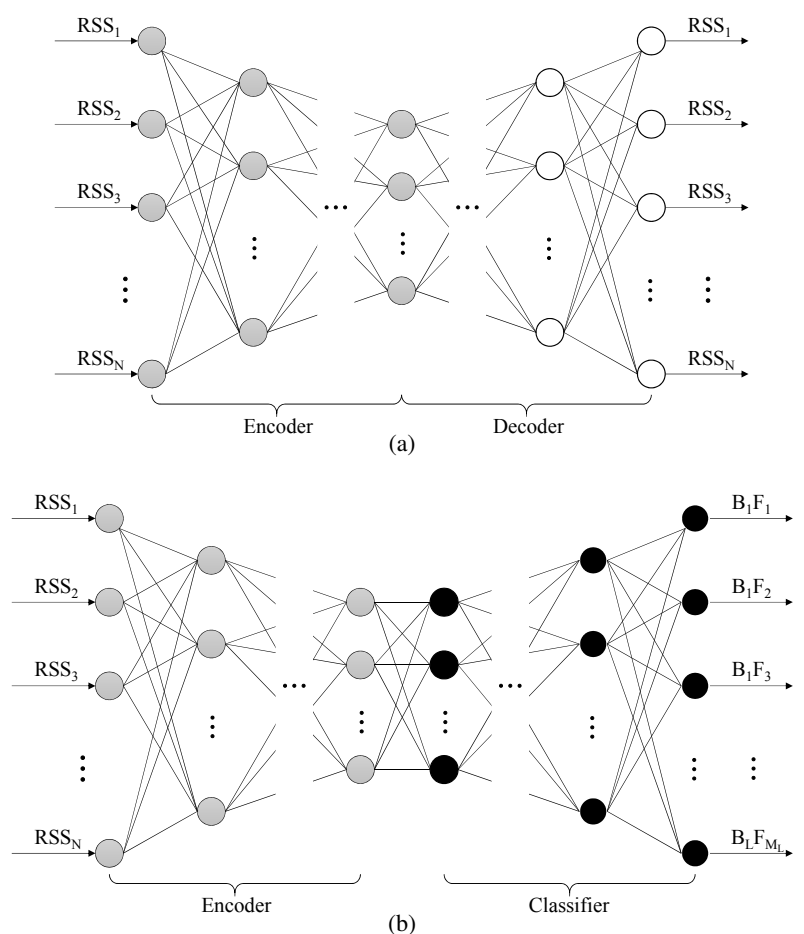

Fig. 2. A DNN architectures for building/floor classification: (a) A stacked autoencoder (SAE) for the reduction of feature space dimension; (b) an overal architecture consisting of the encoder part of the SAE and a feed-forward classifier for multi-class classification with flattened building-floor labels [6].

which utilizes RSSs from Wi-Fi APs to estimate a user location: As shown in Fig. 1 (a), RSSs from nearby APs are submitted by a user or an App running background in the user's mobile device to the system as part of a service request. The submitted RSS measurements, then, are compared with the RSS samples of known locations (i.e., location fingerprints) stored in the database at the fingerprinting server to find the closest match; the location ID of the closest match (e.g., "EB306, 1 in is returned as the user's estimated location. Based on the estimated location, the system provides location-aware services by integrating existing data and services available on XJTLU Intranet and tailoring them for the location as shown in Fig. 1 (b).

\section{HierarchicAl ClassificAtion of BUILDING AND Floor using A Multi-LABEl Classifier}

In [6], the authors proposed a DNN system for building/floor classification that uses a stacked autoencoder (SAE) before a feed-forward classifier to reduce the dimension of the feature space for robust and precise classification as shown in Fig. 2 Note that, after training with RSSs as both input and output data, only the gray-colored nodes are used as an encoder for feature space dimension reduction as shown in Fig. 2(b). The performance of the proposed DNN system was verified with the UJIIndoorLoc dataset [3] and is shown to be comparable

\footnotetext{
${ }^{1}$ It means the room 306 on the third floor of the EB building.
} 
to that of the state-of-the-art systems (i.e., about $92 \%$ of combined building and floor recognitions).

The work reported in [6] is the first in applying a DNN to the building/floor classification of large-scale indoor localization and, as such, reveals a couple of areas of further improvement as follows:

First, the DNN architecture is not optimized enough. While they achieve $92 \%$ accuracy in building/floor classification with an SAE (i.e., only the encoder part) containing three hidden layers of 256, 128, and 64 neurons and a feed-forward classifier with two hidden layers of 128 neurons per each, we could achieve a nearly equivalent performance with an SAE having just two hidden layers of 64 and 4 neurons and a classifier without any hidden layer (i.e., the output layer of the SAE is directly connected to the system output layer).

Second, the proposed DNN system in [6] does not take into account the hierarchical nature of the classification problem at hand due to its calculating the loss and the accuracy over flattened building-floor labels (e.g., $\left(\mathrm{B}_{\mathrm{i}}, \mathrm{F}_{\mathrm{j}}\right) \rightarrow$ " $\mathrm{B}_{\mathrm{i}}-\mathrm{F}_{\mathrm{j}}$ "” In other words, the misclassification of building and that of floor have equal loss during the training phase and results in the same accuracy during the evaluation phase.

To take into account the hierarchical nature of the building/floor classification, we are now considering two possible approaches: One approach is the use of a hierarchical loss function (e.g., a loss function with different weights for building and floor) with the existing multi-class classifier DNN architecture and flattened labels. The other is the use of multilabel classification with arg max functions to convert results back to those of multi-class classification 3

As for the first approach, we found that the hierarchical loss function for flattened labels does not provide a welldefined gradient function, which forces us to use evolutionary algorithms (e.g., genetic algorithm (GA) [8] and particle swarm optimization (PSO) [9]) for training of DNN weights. Due to its many tradeoffs between complexity and flexibility resulting from the use of evolutionary algorithms in DNN weight training, this approach could be an interesting topic for long-term research.

In case of the use of the multi-label classification, applying different weights to building and floor labels during the training phase is rather straightforward, and it is also easy to convert the results of multi-label classification into those of multi-class classification using arg max functions. Fig. 3 shows a newly proposed hierarchical building/floor classifier based on multi-label classification framework with arg max functions.

The classification of building and floor with the proposed architecture shown in Fig. 3 is achieved as follows: The three building and five floor identifiers in the UJIIndoorLoc dataset are one-hot encoded into an eight-dimensional vector (e.g.,

\footnotetext{
${ }^{2} \mathrm{~B}_{\mathrm{i}}$ and $\mathrm{F}_{\mathrm{j}}$ denote building and floor labels, respectively.

${ }^{3}$ In multi-class classification (also called single-label classification), an instance is associated with only a single label from a set of disjoint labels; in multi-label classification, on the other hand, an instance may be associated with multiple labels [7|.
}

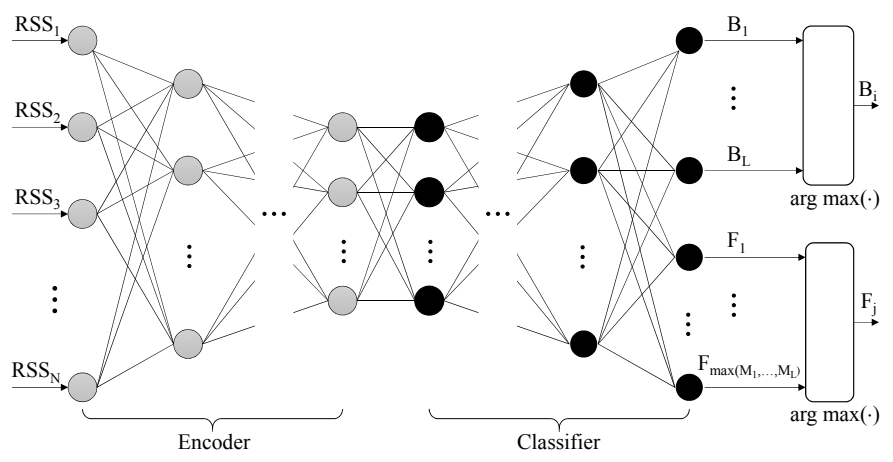

Fig. 3. A DNN architectures for hierarchical building/floor classification based on an SAE for the reduction of feature space dimension and a feed-forward classifier for multi-label classification.

TABLE I

PRELIMINARY RESUlts OF HIERARCHICAL BUILding/FloOR ClassificATION

\begin{tabular}{|r|r|c|c|c|}
\hline \multicolumn{2}{|c|}{ Class Weight } & \multicolumn{3}{|c|}{ Accuracy } \\
\hline Building & Floor & Overall & Building & Floor \\
\hline \hline 1 & 1 & $8.991899 \mathrm{e}-01$ & $9.918992 \mathrm{e}-01$ & $9.036904 \mathrm{e}-01$ \\
\hline 2 & 1 & $9.063906 \mathrm{e}-01$ & $9.918992 \mathrm{e}-01$ & $9.090909 \mathrm{e}-01$ \\
\hline 5 & 1 & $9.207921 \mathrm{e}-01$ & $9.972997 \mathrm{e}-01$ & $9.234923 \mathrm{e}-01$ \\
\hline $\mathbf{1 0}$ & $\mathbf{1}$ & $\mathbf{9 . 3 3 3 9 3 3 e - 0 1}$ & $\mathbf{9 . 9 7 2 9 9 7 e - 0 1}$ & $\mathbf{9 . 3 4 2 9 3 4 e - 0 1}$ \\
\hline 20 & 1 & $9.216922 \mathrm{e}-01$ & $9.936994 \mathrm{e}-01$ & $9.225923 \mathrm{e}-01$ \\
\hline
\end{tabular}

"001|01000" for the third building and the second floor within it) and classified with different class weights for buildings (i.e., for the first three digits) and for floors (i.e., for the last five digits); the one-hot-encoded vector from the multi-label classifier is split into a three-dimensional building and a fivedimensional floor vectors, and the index of a maximum value of each vector is returned as a classified class by the arg max function.

The advantages of the proposed building/floor classifier is two-fold: First, the number of output nodes is smaller compared to that of [6]: In case of the UJIIndoorLoc dataset, the number of output nodes is eight (i.e., the number of buildings (3) plus the maximum of the numbers of floors for buildings (5)), while that of [6] is thirteen; the difference could be much larger for large-scale access where there are lots of buildings (i.e., $\gg 3$ ) with many floors.

Second, as described, the allocation of class weights can be done separately for buildings and floors, which provides more degrees of freedom in classification ${ }^{4}$ In this regard, we are investigating the dependence of building and floor accuracies as well as overall accuracy on class weights through extensive experiments. Table 1 shows preliminary results, and Table II summarizes common DNN parameter values for those experiments.

According to the results shown in Table I] the class weights of 10 and 1 for buildings and floors provide the best results, but the dependence of accuracies on the class weights seems

\footnotetext{
${ }^{4}$ Note that the class weights are originally for cost adjustment for imbalanced data, not for hierarchical classification.
} 
TABLE II

DNN PARAMETER VALUES FOR HIERARCHICAL BUILDING/FLOOR CLASSIFICATION

\begin{tabular}{|l|l|}
\hline \multicolumn{1}{|c|}{ DNN Parameter } & \multicolumn{1}{c|}{ Value } \\
\hline \hline Ratio of Training Data to Overall Data & 0.70 \\
Number of Epochs & 20 \\
Batch Size & 10 \\
\hline SAE Hidden Layers & $64-8-64$ \\
SAE Activation & Rectified Linear (ReLU) \\
SAE Optimizer & ADAM [10] \\
SAE Loss & Mean Squared Error (MSE) \\
\hline Classifier Hidden Layers & None \\
Classifier Optimizer & ADAM \\
Classifier Loss & Binary Crossentropy \\
Classifier Dropout Rate & 0.20 \\
\hline
\end{tabular}

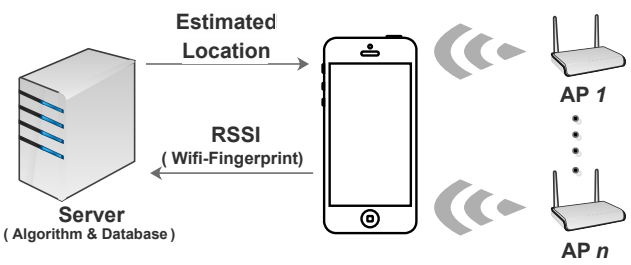

Fig. 4. A prototype of DNN-based indoor localization system for floor-leve location estimation.

not to be big. This suggests that we need to carry out a more systematic investigation on this before making any meaningful conclusions.

\section{DEMONSTRATION OF A DNN-BASED INDOOR LOCALIZATION SYSTEM FOR FLOOR-LEVEL LOCATION ESTIMATION}

To show the feasibility of DNN-based indoor localization, we have implemented a prototype shown in Fig. 4 and demonstrated floor-level location estimation with real RSS data measured on the fourth floor of the EE building on the north campus of XJTLU.

The fingerprinting server is implemented using Flask [11], a Python-based microframework for web development, with SQLite [12] database engine; we chose Flask because we also used Python-based deep learning frameworks, i.e., keras [13] and TensorFlow [14], for the implementation of DNN-based localization algorithms, which are nearly identical to the one used for building/floor classification in [6]. When a client, i.e., an Android App running on a user's mobile phone, submits scanned RSSs, the server estimates its location based on the implemented localization algorithm and returns the estimated location back to the client.

Fig. IV] shows a floor layout of the fourth floor of the EE building where Wi-Fi fingerprints were collected. The areas highlighted in yellow are part of seven locations selected to collect data and test the prototype; both isolated rooms and open public spaces are considered. During the measurement, we used Android mobile phones from different brands to take into account device dependency of Wi-Fi fingerprints.

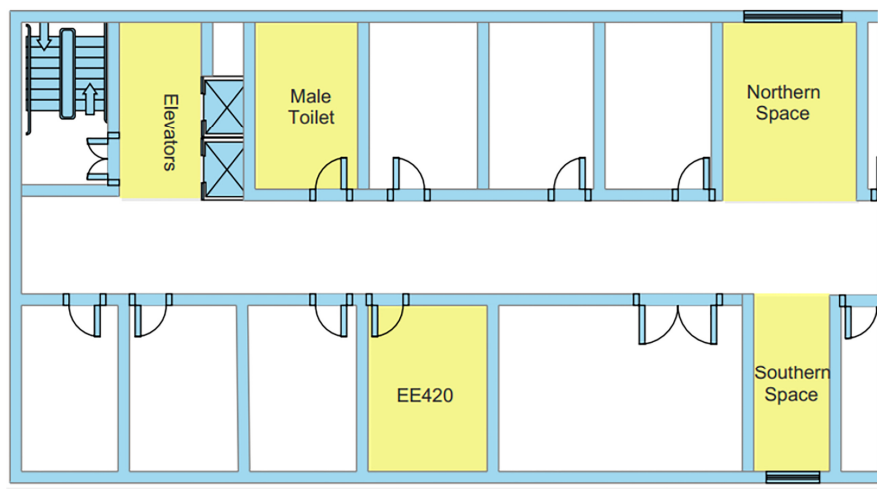

Fig. 5. A partial layout of the fourth floor of the EE building on the north campus of XJTLU.

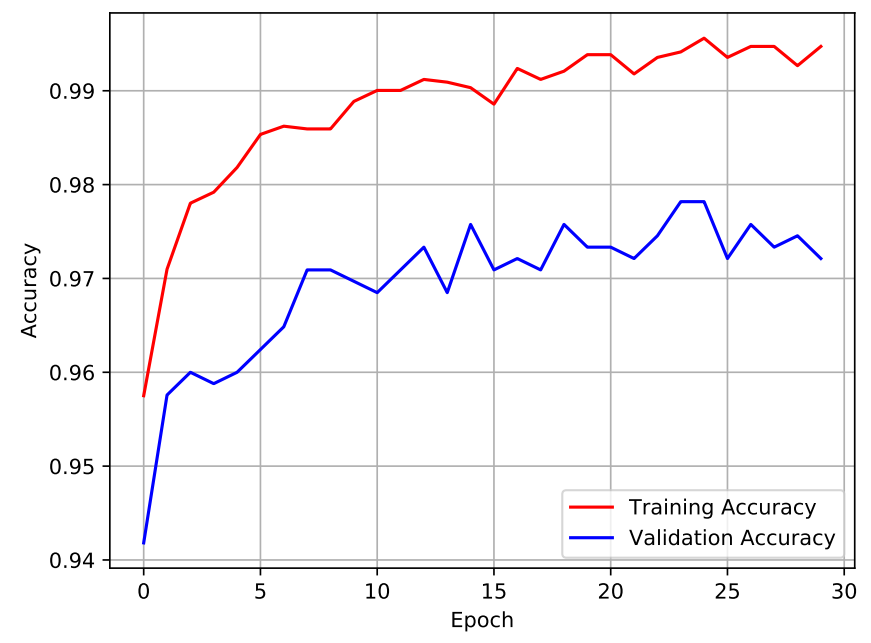

Fig. 6. Training and validation accuracy of floor-level location estimation by the DNN-based indoor localization system with real RSS data.

From around 200 APs detected, we collected more than 4000 fingerprints at different locations with labels.

Fig. 6 shows the training and validation accuracy of floorlevel location estimation by the DNN-based indoor localization system with the collected RSS dataset. As shown in the figure, the DNN architecture used for building/floor classification in [6] can achieve more than $97 \%$ accuracies after thirteenth epoch in floor-level localization with both training and validation datasets. After training and validation, we applied the system to a training dataset of 253 fingerprints and obtained the accuracy of 0.97198 , which is the average of five trials. Note that, even though the testing accuracy is pretty high, it may be lower if we test with a much larger dataset.

\section{Summary}

In this paper we have introduced the feasibility study project on the XJTLU Campus Information and Visitor Service system, which will serve as a test bed for large-scale locationaware services in access, and reported preliminary results of our investigation on the use of DNNs for building/floor classi- 
TABLE III

DNN PARAmeter VALUES FOR FlOOR-LeVEl Location Estimation

\begin{tabular}{|l|l|}
\hline \multicolumn{1}{|c|}{ DNN Parameter } & \multicolumn{1}{c|}{ Value } \\
\hline \hline Ratio of Training Data to Overall Data & 0.75 \\
Batch Size & 10 \\
\hline SAE Hidden Layers & $128-64-32-64-128$ \\
SAE Activation & Hyperbolic Tangent (TanH) \\
SAE Optimizer & ADAM \\
SAE Loss & MSE \\
\hline Classifier Hidden Layers & $64-32-7$ \\
Classifier Activation & ReLU \\
Classifier Optimizer & AdaGrad [15] \\
Classifier Loss & Cross Entropy \\
Classifier Dropout Rate & 0.50 \\
Classifier Epochs & 30 \\
\hline
\end{tabular}

fication and floor-level location estimation. The preliminary results for both building/floor classification and floor-level location estimation clearly show the strengths of DNN-based approaches, including immunity against signal fluctuation, noise effects, and device dependency, no need of finding the best match against every fingerprint in a database after DNN training, and the elimination of time-consuming manual parameter tuning.

Still, further study is needed for hierarchical building/floor classification and more scalable \& higher resolution floor-level localization.

Note that the implemented DNN models, results of performance evaluation, and collected RSS datasets are available online at the project home page: http://kyeongsoo.github.io/ research/projects/indoor_localization/index.html

\section{ACKNOWLEDGMENT}

This work was supported in part by Xi'an JiaotongLiverpool University (XJTLU) Research Development Fund (under Grant RDF-14-01-25), Summer Undergraduate Research Fellowships programme (under Grant SURF201739), Research Institute for Smart and Green Cities Seed
Grant Programme 2016-2017 (under Grant RISGC-2017-4), and Centre for Smart Grid and Information Convergence.

\section{REFERENCES}

[1] P. Bahl and V. N. Padmanabhan, "RADAR: An in-building RF-based user location and tracking system," in Proc. 2000 IEEE INFOCOM, vol. 2, 2000, pp. 775-784.

[2] A. Moreira, M. J. Nicolau, F. Meneses, and A. Costa, "Wi-Fi fingerprinting in the real world - RTLSUM at the EvAAL competition," in Proc. International Conference on Indoor Positioning and Indoor Navigation (IPIN), Banff, Alberta, Canada, Oct. 2015, pp. 1-10.

[3] J. Torres-Sospedra, R. Montoliu, A. Martínez-Usó, J. P. Avariento, T. J. Arnau, M. Benedito-Bordonau, and J. Huerta, "UJIIndoorLoc: A new multi-building and multi-floor database for wlan fingerprint-based indoor localization problems," in Proc. International Conference on Indoor Positioning and Indoor Navigation (IPIN), Busan, Korea, Oct. 2014, pp. 261-270.

[4] G. Félix, M. Siller, and E. N. Álvarez, "A fingerprinting indoor localization algorithm based deep learning," in Proc. ICUFN 2016, Vienna, Austria, Jul. 2016, pp. 1006-1011.

[5] W. Zhang, K. Liu, W. Zhang, Y. Zhang, and J. Gu, "Deep neural networks for wireless localization in indoor and outdoor environments," Neurocomputing, vol. 194, pp. 279-287, Jun. 2016.

[6] M. Nowicki and J. Wietrzykowski, "Low-effort place recognition with wifi fingerprints using deep learning," ArXiv e-prints, Apr. 2017, arXiv:1611.02049v2 [cs.RO]. [Online]. Available: https://arxiv.org/abs/ $1611.02049 \mathrm{v} 2$

[7] G. Tsoumakas and I. Katakis, "Multi-label classification: An overview," International Journal of Data Warehousing \& Mining, vol. 3, no. 3, pp. 1-13, 2007.

[8] D. E. Goldberg, Genetic Algorithms in Search, Optimization and Machine Learning. Reading, Massachusetts: Addison-Wesley, 1989.

[9] J. Kennedy and R. Eberhart, "Particle swarm optimization," in Proc. IEEE International Conference on Neural Networks. IV., Perth, WA, Australia, 1995, pp. 1942-1948.

[10] D. Kingma and J. Ba, "ADAM: A method for stochastic optimization," ArXiv e-prints, Jan. 2017, arXiv:1412.6980 8 [cs.LG]. [Online]. Available: https://arxiv.org/abs/1412.6980v8

[11] Flask. [Online]. Available: http://flask.pocoo.org/

[12] SQLite. [Online]. Available: https://www.sqlite.org/

[13] Keras: The Python deep learning library. [Online]. Available: https://keras.io/

[14] TensorFlow ${ }^{\overline{1 M}}$. [Online]. Available: https://www.tensorflow.org/

[15] J. Duchi, E. Hazan, and Y. Singer, "Adaptive subgradient methods for online learning and stochastic optimization," Journal of Machine Learning Research, vol. 12, pp. 2121-2159, 2011. 